\title{
Análisis de la influencia del Índice de Masa Corporal en la evolución de la Insuficiencia Cardíaca en una Zona de Salud
}

\author{
Pedro J Tarraga Lopez ${ }^{a, *}$ \\ a Departamento de Atención Primaria, Medicina de Familia y Comunitaria, Facultad de Medicina de Albacete, \\ Universidad de Castilla-La Mancha, Albacete, España. \\ *pedrojuan.tarraga@uclm.es
}

Editor Asignado: José Miguel Soriano del Castillo. Universidad de Valencia. Valencia, España.

Recibido el 23 de agosto de 2019; aceptado el 19 de abril de 2020; publicado el 5 de junio de 2020.

\section{PALABRAS CLAVE}

Obesidad;

Índice de Masa

Corporal;

Insuficiencia

Cardíaca;

Atención Primaria de Salud.

Análisis de la influencia del Índice de Masa Corporal en la evolución de la Insuficiencia Cardíaca en una Zona de Salud

\section{RESUMEN}

Introducción: Analizar las diferentes variables del Índice de Masa Corporal (IMC) en relación con la evolución de la Insuficiencia cardíaca en una Zona de Salud.

Material y Métodos: Estudio descriptivo observacional y analítico de los 161 pacientes diagnosticados en la Zona de Salud entre enero de 2014 y diciembre de 2016. Se analizaron datos demográficos, clínicos, y analíticos. Se calculó el IMC a partir del peso y la talla en la primera visita a la consulta, mediante la fórmula: peso $(\mathrm{kg})$ / cuadrado de la talla (m). Una vez obtenido se evaluó la relación entre el IMC y la evolución en 2 años. Se estudió a 4 subgrupos de pacientes, en función de su IMC, a partir de los criterios definidos por la Organización Mundial de la Salud (OMS) en 1999: bajo peso (IMC<20,5), peso normal (IMC de 20,5 a <25,5), sobrepeso (IMC de 25,5 a $<30$ ) y obesidad (IMC $\geq 30$ ).

Resultados: 81 participantes eran obesos (50,8\%), 33 hombres y 48 mujeres. La edad media de los obesos fue de $80,32(9,23)$ años. Un $62,2 \%$ tenía diagnosticado algún tipo de cardiopatía, siendo: $29,2 \%$ cardiopatía isquémica; $46,6 \%$ arritmias cardiacas y $20,5 \%$ valvulopatías. El IMC, como variable continua, se asoció de forma significativa con la mortalidad ( $p<0,001)$, la edad $(0,002)$, la enfermedad isquémica $(0,001)$, sexo $(0,004)$, hipertensión $(0,002)$, diabetes $(0,003)$ y dislipemia $(0,004)$. También se vio relación directamente proporcional del IMC con el uso de tratamientos con digoxina, diuréticos de asa y espironolactona. EL IMC también se asoció con el número de ingresos, mayor número de enfermedades crónicas concomitantes y mortalidad. Los pacientes con obesidad obtuvieron peores puntuaciones en el cuestionario de calidad de vida MLWHFQ.

Conclusiones: EI IMC mostró asociación con la mortalidad, la enfermedad isquémica, el sexo, la hipertensión, diabetes y dislipemia en pacientes con insuficiencia cardiaca. 
Analysis of the influence of Body Mass Index in the evolution of Heart Failure in a Health Area

\section{KEYWORDS}

Obesity;

Body Mass Index;

Heart Failure;

Primary Health

Care.

\section{ABSTRACT}

Introduction: To analyze the relationship of the Body Mass Index (BMI) with heart failure in a Health Area.

Material and Methods: Observational descriptive study of the 161 patients who had been diagnosed in the Health Area between January 2014 and December 2016. Demographic, clinical, and analytical data have been analyzed. The BMI is calculated from the weight and height on the first visit to the consultation, using the formula: weight (in kilograms) / square of height (in meters). Once obtained, the relationship between BMI and evolution in 2 years was evaluated. Four subgroups of patients are studied, based on their BMI, based on the criteria defined by the World Health Organization (WHO) in 1999: low weight (BMI<20.5), normal weight (BMI 20.5 to $<25.5$ ), overweight (BMI 25.5 to $<30$ ) and obesity (BMI $\geq 30$ ).

Results: Of the participants, 81 were obese (50.8\%), being 33 men and 48 women. The average age of the obese is 80.32 (9.23) years. The main causes of heart failure in $62.2 \%$ had diagnosed some type of heart disease, being: $29.2 \%$ ischemic heart disease, $46.6 \%$ cardiac arrhythmias and $20.5 \%$ valvulopathies. BMI as a continuous variable was significantly associated with mortality $(p<0.001)$, age (0.002), ischemic disease (0.001), gender (0.004), hypertension (0.002), diabetes (0.003) and dyslipidemia (0.004). The relation of BMI with the use of Digoxin, Asa Diuretics and Spironolactone treatments has also been seen with higher BMI plus utilization. BMl is also associated with the number of admissions, greater number of concomitant chronic diseases and mortality. Obese patients scored higher on the MLWHFQ quality of life questionnaire, which corresponds to a poorer quality of life.

Conclusions: BMI has been shown to be associated with mortality, ischemic disease, sex, hypertension, diabetes and dyslipidemia in patients with heart failure.

\section{CITA}

\section{INTRODUCCIÓN}

Los cambios en la dieta y el aumento del sedentarismo progresivo y generalizado propios de nuestros tiempos han conllevado un aumento progresivo de la incidencia y la prevalencia de la obesidad en la población general ${ }^{1}$. Este incremento tiene una distribución geográfica heterogénea y afecta principalmente a los países occidentales; tanto Estados Unidos² como Europa están siendo víctimas de esta epidemia. Según el Registro de la Sociedad Española para el Estudio de la Obesidad (SEEDO) ${ }^{3}$, entre 1999 y 2000, la prevalencia de obesidad (índice de masa corporal $[\mathrm{IMC}] \geq 30$ ) en la población española adulta era del $14,5 \%$, predominantemente entre las mujeres, y se incrementaba con la edad, hasta un 20-30\% de los mayores de 55 años.

La obesidad es un conocido factor de riesgo independiente de insuficiencia cardiaca (IC) ${ }^{1}$ que ha alcanzado proporciones epidémicas: la Organización Mundial de la Salud calcula que más de mil millones de adultos en todo el mundo tienen sobrepeso y, de ellos, 300 millones son clínicamente obesos. Tanto la incidencia como la prevalencia de la obesidad y la insuficiencia cardíaca son tan elevadas que no es raro encontrar ambos trastornos en un mismo paciente. De hecho, varias cohortes de pacientes con insuficiencia cardíaca han revelado que un $15-35 \%$ de dichos pacientes son obesos y que un $30-60 \%$ tienen problemas de sobrepeso 2 . 
Los estudios epidemiológicos han mostrado claramente una estrecha relación entre la obesidad y mayor riesgo de enfermedades cardiovasculares (ECV) y mortalidad en la población general.

Descubrir la relación entre la obesidad y la insuficiencia cardíaca está resultando complejo; un estudio epidemiológico reciente, derivado del Framingham Heart Study, indica claramente que la obesidad y el sobrepeso son variables muy predictivas de una posterior insuficiencia cardíaca clínica ${ }^{1}$. Aunque la obesidad causa anormalidades en la función diastólica y sistólica, y se supone que aumenta el riesgo de mortalidad en los pacientes con insuficiencia cardíaca establecida, nuestro grupo y otros han mostrado que, paradójicamente, el IMC está en relación inversa con la mortalidad a largo plazo en pacientes con insuficiencia cardíaca cróni$\mathrm{ca}^{2,4,5-13}$. Sin embargo, es importante observar que el IMC no es el único factor convencional de riesgo de ECV que presenta una asociación paradójica en los desenlaces clínicos de pacientes con insuficiencia cardíaca. Las concentraciones elevadas de lipoproteínas de baja densidad, así como del colesterol total, se han asociado también con una ventaja de supervivencia en la insuficiencia cardíaca. Estos hallazgos sistemáticos en diversos factores de riesgo de la ECV en los pacientes con insuficiencia cardíaca justifican el empleo del término epidemiología inversa ${ }^{12-18}$.

El objetivo fue analizar la relación entre el IMC y la evolución de la Insuficiencia Cardíaca durante un seguimiento a 2 años en pacientes con insuficiencia cardíaca atendidos de forma ambulatoria en nuestra Zona de Salud por médicos y enfermeras de Atención Primaria, valorando si esta relación se afectaba por el número de reingresos hospitalarios $y$, finalmente, valorar si el IMC tiene influencia en la calidad de vida, pues en las publicaciones hay discrepancias al respecto.

\section{MATERIAL Y MÉTODOS}

Estudio descriptivo observacional y analítico de los 161 pacientes que habían sido diagnosticados de I I ${ }^{12,13}$ en la Zona de Salud con una población mayor de 14 años de 15.000 habitantes, entre enero de 2014 y diciembre de 2016. Se analizó a los que se disponía del IMC en la primera visita y de su situación vital a los 2 años de seguimiento. El criterio de inclusión en estudio fue la insuficiencia cardíaca como diagnóstico principal del paciente. Se estudiaron datos demográficos, clínicos, y analíticos, se calculó el IMC a partir del peso y la talla en la primera visita a la consulta, mediante la fórmula: peso (kg) / cuadrado de la talla (m), posteriormente se evaluó la relación entre el IMC y la supervivencia a 2 años. Se clasificaron 4 subgrupos de pacientes, en función de su IMC, a partir de los criterios definidos por la Organización Mundial de la Salud (OMS) en 199919: bajo peso (IMC $<0,5)$, peso normal (IMC de 20,5 a $<25,5)$, sobrepeso (IMC de 25,5 a $<30$ ) y obesidad (IMC $\geq 30$ ).

El análisis estadístico se realizó mediante el paquete estadístico SPSS ${ }^{\circledR} 24.0$ para Windows. La asociación entre el IMC como variable continua y la mortalidad a 2 años se analizó mediante la prueba de la U de Mann-Whitney y el test de Kruskal-Wallis para los que no tenía distribución normal. Para el cálculo de la odds ratio (OR) se utilizó la regresión logística. En el análisis multivariable de regresión logística ${ }^{16}$ se introdujo la mortalidad y los reingresos a 1 y 2 años como variable dependiente, y como variables independientes, el IMC (como variable continua), la edad, el sexo, la etiología de la insuficiencia cardíaca, la presencia de diabetes e hipertensión arterial y los tratamientos recibidos (bloqueadores beta, inhibidores de la enzima de conversión de angiotensina [IECA] o antagonistas de los receptores de la angiotensina II [ARA-II], diuréticos de asa, espironolactona, digoxina y estatinas). El método utilizado fue «por pasos hacia atrás condicional».

La relación entre los diferentes grupos establecidos de IMC con los reingresos y la mortalidad a 1 y 2 años se analizó mediante la prueba de la $\chi^{2}$ (asociación lineal por lineal para el análisis conjunto de los cuatro grupos) o mediante el test de Fisher, en función del número de pacientes. Las comparaciones entre grupos se realizaron mediante la prueba de la $\chi^{2}$ para las variables categóricas y el test de Kruskal-Wallis para las variables continuas, tras comprobar que no tenían distribución normal.

Se evaluó la calidad de vida de los pacientes mediante el Minnesota Living With Heart Failure Questionnaire (MLWHFQ) ${ }^{12}$, ya utilizado previamente en España ${ }^{13}$, durante la visita de inclusión en el estudio.

EI MLWHFQ se compone de 21 preguntas cuyo objetivo es averiguar en qué medida la insuficiencia cardíaca afecta a los aspectos físico, psíquico y socioeconómico de la vida de los pacientes; las preguntas se refieren a signos y síntomas de insuficiencia cardíaca, relaciones sociales, actividad física y sexual, trabajo y emociones; la gama de respuestas posibles para cada pregunta va de 0 (no) a 5 (muchísimo), de manera que a mayor puntuación peor calidad de vida.

El estudio se realizó cumpliendo la ley de protección de datos personales y de acuerdo con las recomendaciones internacionales sobre investigación clínica de la Declaración de Helsinki de la Asociación Médica Mundial. 


\section{RESULTADOS}

Participaron 161 pacientes diagnosticados de IC en nuestra Zona de Salud. Edad media 81,24 (9,59) años (edad media/ desviación estándar), fueron mujeres un $54 \%$. De los participantes, 81 eran obesos $(50,8 \%)$, siendo 33 hombres y 48 mujeres. La edad media de los obesos fue de 80,32 $(9,23)$ años. Las principales causas de insuficiencia cardíaca en un $62,2 \%$ tenían diagnosticado algún tipo de cardiopatía, siendo: $29,2 \%$ cardiopatía isquémica, $46,6 \%$ arritmias cardíacas y $20,5 \%$ valvulopatías. En las Tablas 1 y 2 se muestran las características clínicas y los tratamientos recibidos por los pacientes.

La mortalidad a los 2 años de seguimiento fue del 19,1\%, se aprecia que en pacientes con sobrepeso y obesidad hay aumento de mortalidad con significación estadística $(P<0,001)$.
EI IMC como variable continua se asoció de forma significativa con la mortalidad $(p<0,001)$, la edad $(0,002)$, la enfermedad isquémica $(0,001)$, sexo $(0,004)$, hipertensión arterial (HTA) $(0,002)$, diabetes $(0,003)$ y dislipemia $(0,004)$ (Tabla 1). También se ha visto relación del IMC con el uso de tratamientos con digoxina, diuréticos de asa y espironolactona a mayor IMC mas utilización (Tabla 2).

EL IMC también está asociado con el número de ingresos, mayor número de enfermedades crónicas concomitantes y mortalidad (Tabla 3).

Tras ajustar por edad, sexo, etiología, hipertensión, diabetes y los distintos tratamientos recibidos, el IMC se mantuvo como predictor independiente de insuficiencia cardíaca $(\mathrm{OR}=0,92[0,88-0,97])$ (Tabla 4), lo que quiere decir, que tras el ajuste, el riesgo de insuficiencia cardíaca por cualquier causa es mayor un $8 \%$ por cada aumento de 1 punto en el IMC.

Tabla 1. Características clínicas en función del índice de masa corporal.

\begin{tabular}{|c|c|c|c|c|c|}
\hline & $\begin{array}{c}\text { IMC } \\
(<18,5)\end{array}$ & $\begin{array}{c}\text { IMC1 } \\
(18,5-25)\end{array}$ & $\begin{array}{c}\text { IMC2 } \\
(25,1-30)\end{array}$ & $\begin{array}{c}\text { IMC3 } \\
(>30,1)\end{array}$ & 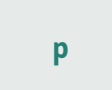 \\
\hline Edad & $67,4(6,2)$ & $73,2(7,1)$ & $82,6(5,8)$ & $84,5(6,7)$ & $<0,002$ \\
\hline Hombres & $80 \%$ & $65 \%$ & $45 \%$ & $32 \%$ & $<0,004$ \\
\hline Causa Isquémica & $27,5 \%$ & $64,5 \%$ & $57 \%$ & $48 \%$ & $<0,001$ \\
\hline HTA & $11 \%$ & $18 \%$ & $30 \%$ & $41 \%$ & $<0,002$ \\
\hline Diabetes & $12 \%$ & $13 \%$ & $28 \%$ & $47 \%$ & $<0,003$ \\
\hline Dislipemia & $16 \%$ & $18 \%$ & $30 \%$ & $44 \%$ & $<0,004$ \\
\hline Tabaquismo & $20 \%$ & $29 \%$ & $25 \%$ & $26 \%$ & NS \\
\hline
\end{tabular}

IMC: índice de masa corporal; HTA: hipertensión arterial.

Tabla 2. Tratamientos en función del índice de masa corporal (no absoluto).

\begin{tabular}{|c|c|c|c|c|c|}
\hline & Bajo Peso & Peso Normal & Sobrepeso & Obesidad & $p$ \\
\hline Bloqueadores beta & 14 & 16 & 46 & 24 & NS \\
\hline IECA o ARA II & 8 & 14 & 31 & 47 & NS \\
\hline Espironolactona & 5 & 12 & 34 & 49 & $<0,002$ \\
\hline Digoxina & 0 & 0 & 45 & 55 & $<0,006$ \\
\hline Diuréticos asa & 14 & 10 & 23 & 53 & $<0,001$ \\
\hline Estatinas & 10 & 21 & 34 & 35 & NS \\
\hline
\end{tabular}


Tabla 3. Ingresos, mortalidad, causas y enfermedades crónicas.

\begin{tabular}{l|c|c|c|c|c}
\hline Ingresos Hospital en 1 año & Bajo Peso & Peso Normal & Sobrepeso & Obesidad & P \\
\hline Ingresos Hospital en 3 años & $8 \%$ & $12 \%$ & $42 \%$ & $48 \%$ & $<0,001$ \\
\hline $\begin{array}{l}\text { Mortalidad } \\
\text { Enfermedades crónicas }\end{array}$ & $7,5 \%$ & $11,5 \%$ & $30 \%$ & $51 \%$ & $<0,003$ \\
concomitantes & 0 & $7 \%$ & $47 \%$ & $46 \%$ & $<0,001$ \\
\hline $\begin{array}{l}\text { Infección respiratoria } \\
\text { como causa de ingreso }\end{array}$ & $5 \%$ & $8 \%$ & $25 \%$ & $62 \%$ & $<0,001$ \\
\hline $\begin{array}{l}\text { Descompensación cardíaca } \\
\text { como causa de Ingreso }\end{array}$ & 0 & $8 \%$ & $22 \%$ & 0 & NS \\
\hline
\end{tabular}

Las puntuaciones medias obtenidas en el cuestionario de calidad de vida MLWHFQ fueron: 23 puntos para bajo peso; 21 puntos para normopeso; 34 puntos para sobrepeso; 43 puntos para obesidad. Los pacientes con obesidad obtuvieron puntuaciones más altas de inicio y final, que corresponde a una peor calidad de vida. Hay diferencias significativas entre las puntuaciones obtenidas por los pacientes de peso normal, con sobrepeso y obesidad ( $p<0,03$ y 0,002 , respectivamente), mostrando estos peor calidad de vida que los de peso normal o bajo peso.

\section{DISCUSIÓN}

La obesidad es un factor de riesgo cardiovascular común y frecuentemente ignorado por los médicos. La obesidad se asocia a varias enfermedades cardiovasculares y está vinculada no sólo a enfermedad coronaria, sino también a alteraciones del ritmo cardiaco y la función ventricular. Esta asociación se da por múltiples mecanismos, y no sólo a través de la hipertensión, la diabetes mellitus o la dislipemia. El diagnóstico de obesidad debe incluir mediciones de contenido total y de distribución de la grasa corporal. Aunque el manejo de la obesidad es difícil, el manejo integral de la obesidad puede resultar favorable ${ }^{1-4,12,13,17,18,20}$.

La obesidad mórbida es un factor de riesgo conocido de insuficiencia cardíaca. Se desconoce, sin embargo, si el sobrepeso y los grados menores de obesidad también son factores de riesgo de IC.

En este estudio se aprecia una incidencia de IC del 1\%, que si bien esta en concordancia con otros estudios publicados, son cifras inferiores a otros estudios nacionales que refieren incidencias del $5 \%$, pudiéndose justificar esta diferencia por un infra diagnóstico o mala codificación diagnóstica en la historia clínica ${ }^{1-3}$.

El presente estudio investiga la relación entre el índice de masa corporal y la incidencia de IC en los pacientes de una Zona de Salud. Durante el periodo de seguimiento (que fue de 2 años de media), se observó una incidencia de IC de 161 pacientes, siendo obesos 81 de los participantes, 33 hombres y 48 mujeres. La edad media de los obesos es de 80,32 años, mientras que en los no obesos era de 81,24 años. Tras el ajuste por los factores de riesgo de IC establecidos, se observó que existía un incremento del riesgo de IC del 5\%

Tabla 4. Análisis multivariable de regresión logística (por pasos hacia atrás condicional).

\begin{tabular}{|l|c:c}
\hline $\begin{array}{l}\text { Variables que } \\
\text { permanecen en el modelo }\end{array}$ & OR & IC95\% \\
\hline Edad (años) & 1,35 & $1,21-1,40$ \\
\hline Diabetes & 2,47 & $1,31-3,61$ \\
\hline IMC & 0,92 & $0,88-0,99$ \\
\hline Bloqueadores beta & 0,64 & $0,25-0,74$ \\
\hline Estatinas & 0,38 & $0,27-0,77$ \\
\hline IECA o ARA-II & 0,39 & $0,25-0,87$ \\
\hline
\end{tabular}

OR: odds ratio; IC95\%: intervalo de confianza del 95\%; IMC: índice de masa corporal;

IECA: inhibidores de la enzima de conversión de angiotensina; ARA-II: antagonistas de los receptores de la angiotensina II. 
en varones y del $10 \%$ en mujeres por cada incremento de 1 en el índice de masa corporal. Comparados con individuos con IMC normal, el riesgo de IC en los pacientes obesos se doblaba. Se apreciaba un aumento gradual del riesgo de IC conforme aumentaba el IMC. Se aprecia que el aumento del índice de masa corporal se asocia con un aumento del riesgo de IC. En vista de la alta prevalencia de la obesidad, las estrategias de promoción de un peso corporal óptimo pueden reducir la incidencia de IC.

Aunque la ratio de riesgo de IC en varones con sobrepeso no alcanzó significación estadística, los análisis evaluando el IMC como una variable continua avalan la existencia de un gradiente continuo de riesgo de IC conforme aumenta el IMC en ambos sexos. Es de destacar el efecto menor del IMC en el riesgo de IC en pacientes con HTA. La falta de efecto del IMC en el riesgo de IC en pacientes con infarto debe interpretarse con cautela dado el pequeño tamaño de la muestra ${ }^{21-22}$.

Tres estudios comunitarios previos también reportaron un aumento del riesgo de IC con el aumento del IMC. La fuerza de la asociación, el aumento gradual del riesgo de IC conforme aumenta el IMC, la secuencia temporal (el aumento del IMC precede al desarrollo de IC) y la consistencia de los resultados en múltiples análisis sugiere una relación causal entre el aumento del IMC y la IC. El aumento de masa corporal es un factor de riesgo de HTA, diabetes mellitus y dislipemia; todos estos factores aumentan el riesgo de infarto de miocardio, una etiología importante de la IC. Además, la HTA y la diabetes aumentan de forma independiente el riesgo de IC. EI IMC aumentado se asocia con un remodelado VI alterado, debido posiblemente al aumento de la sobrecarga hemodinámica, la activación neurohormonal y el aumento del estrés oxidativo. Recientemente se ha postulado la posibilidad de un efecto directo de la obesidad sobre el miocardio al mostrarse esteatosis y lipoapoptosis cardiacas en modelos animales de obesidad ${ }^{23-29}$.

Se presenta una relación estadísticamente significativa entre el número de enfermedades crónicas y los ingresos hospitalarios o mortalidad, lo que confirman otros estudios que hablan de la alta mortalidad y su complejo manejo por la frecuencia de comorbilidades ${ }^{30-32}$.

Un 65\% tienen entre 4 y 6 enfermedades crónicas asociadas observando: HTA (95\%), diabetes (42,2\%), dislipemias $(68,9 \%)$, tabaco $(16,1 \%)$, obesidad $(49,1 \%)$ y neoplasias $(13,7 \%)$. Estos datos confirman el estudio de Nagarajan ${ }^{33}$ en el que un $40 \%$ de pacientes tienen 5 o más problemas de salud asociados que afectan negativamente a su pronóstico, los más importantes son: HTA (55\%), diabetes (31\%) y enfermedad pulmonar obstructiva crónica (26\%), también son frecuentes: hipercolesterolemia, fibrilación auricular, insuficiencia renal, enfermedad cerebrovascular y demencia. Se ha observado en este estudio una reducción del tabaquismo en un $5 \%$.

Mientras que este estudio presenta un 62,2\% de pacientes con diagnóstico de cardiopatía -cardiopatía isquémica $(29,2 \%)$, arritmias cardiacas $(46,6 \%)$ y valvulopatías $(20,5 \%)-$ , otros estudios ${ }^{34-39}$, además de la cardiopatía isquémica y de la HTA, presentan como causas de IC lesiones valvulares o congénitas (10\%) y las miocardiopatías (10\%). El 10\% restante corresponde a arritmias, trastornos de la conducción, estados que cursan con alto gasto cardiaco (anemia, sepsis, tirotoxicosis, Paget), fármacos (algunos quimioterápicos), toxinas (alcohol, cocaína), enfermedades infiltrativas (sarcoidosis, amiloidosis) y diabetes. El tipo de cardiopatía fue distinto entre los grupos siendo la cardiopatía isquémica más frecuente en normopeso y las arritmias más frecuentes en sobrepeso y obesidad, lo que coincide con otros estudios como el de Trullàs ${ }^{4}$ y Cols. realizado en medio hospitalario.

Los tratamientos utilizados están acordes con la fisiopatología de la IC: diuréticos (69,5\%), IECAs (32,9\%), ARASII $(35,4 \%)$, ivabradina (2\%), betabloqueantes $(29,1 \%)$, digoxina (20\%). También se observa alta adherencia terapéutica, un $91,2 \%$ global $^{40-41}$.

Los resultados de este estudio contrastan con los llamados "experiencia de paradoja de la obesidad en pacientes con IC" publicados en España, por Zamora y Cols..$^{40}$ y TruIlàs ${ }^{4}$ que comunicaron su experiencia mediante el análisis retrospectivo el primero y prospectivo el segundo, con la hipótesis de que la obesidad podría ser factor protector de mortalidad, mientras que en nuestro estudio tanto sobrepeso como obesidad son claros factores de empeoramiento de la IC y la mortalidad.

\section{$\longrightarrow$ CONCLUSIONES}

El incremento en el IMC se asoció a mayor mortalidad y mayores ingresos hospitalarios al año junto a un mayor número de enfermedades crónicas que van en detrimento de la calidad de vida.

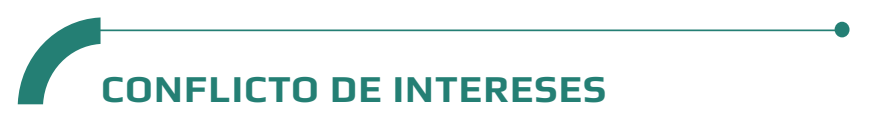

El autor expresa que no existen conflictos de interés al redactar el manuscrito. 


\section{REFERENCIAS}

(1) Rubio MA, Salas J, Barbany M, Moreno B, Aranceta J, Bellido D, et al. Consenso SEEDO 2007 para la evaluación del sobrepeso y la obesidad y el establecimiento de criterios de intervención terapéutica. Rev Esp Obes. 2007; 5: 135-71.

(2) Gustafsson F, Kragelund CB, Torp-Pedersen C, Seibaek M, Burchardt H, Akkan D, et al; and DIAMOND Study Group. Effect of obesity and being overweight on long-term mortality in congestive heart failure: influence of left ventricular systolic function. Eur Heart J. 2005; 26: 58-64.

(3) Aranceta-Bartrina J, Serra-Majem L, Foz-Sala M, MorenoEsteban B; Grupo Colaborativo SEEDO*. Prevalencia de obesidad en España. Med Clin (Barc). 2005; 125(12): 460-6.

(4) Trullàs JC, Formiga F, Montero M, Conde A, Casado J, Carrasco FJ, et al. Paradoja de la obesidad en la insuficiencia cardiaca. Resultados del Registro RICA. Med Clin (Barc). 2011; 137(15): 671-7.

(5) Testa G, Cacciatore F, Galizia G, Della-Morte D, Mazzella F, Langellotto $A$, et al. Waist circumference but not body mass index predicts long-term mortality in elderly subjects with chronic heart failure. ] Am Geriatr Soc. 2010; 58: 1433-40.

(6) Curtis LH, Greiner MA, Hammill BG, Kramer JM, Whellan DJ, Schulman KA, et al. Early and long-term outcomes of heart failure in elderly persons, 2001-2005. Arch Intern Med. 2008; 168: $2481-8$.

(7) Davos CH, Doehner W, Rauchhaus M, Cicoira M, Francis DP, Coats AJ, et al. Body mass and survival in patients with chronic heart failure without cachexia: the importance of obesity. ] Card Fail. 2003; 9: 29-35.

(8) Lavie CJ, Osman AF, Milani RV, Mehra MR. Body composition and prognosis in chronic systolic heart failure: the obesity paradox. Am J Cardiol. 2003; 91: 891-4.

(9) Curtis JP, Selter JG, Wang Y, Rathore SS, Jovin IS, Jadbabaie F, et al. The obesity paradox: body mass index and outcomes in patients with heart failure. Arch Intern Med. 2005; 165: 55-61.

(10) Lavie C], Mehra MR, Milani RV. Obesity and heart failure prognosis: paradox or reverse epidemiology? Eur Heart ]. 2005; 26: 5-7.

(11) Powell BD, Redfield MM, Bybee KA, Freeman WK, Rihal CS. Association of obesity with left ventricular remodeling and diastolic dysfunction in patients without coronary artery disease. Am ] Cardiol. 2006; 98: 116-20.

(12) Dagenais GR, Yi Q, Mann JF, Bosch J, Pogue J, Yusuf S. Prognostic impact of body weight and abdominal obesity in women and men with cardiovascular disease. Am Heart ]. 2005; 149: 54-60.

(13) Tárraga López PJ, Villar Inarejos MJ, Sadek IM, Madrona Marcos F, Tárraga Marcos L, Simón García MA. Quality care in the management of heart failure in a health area. Clin Invest Arterios. 2018: 258-264.

(14) Roldán Santos P, Tarraga Marcos L, Madrona Marcos F, Sadeh IM, Celada Roldan C., Panisello Royo JM, Tárraga López PJ. La telemedicina en la insuficiencia cardíaca, el camino pendiente. JONNPR. 2019; 4(12): 1230-55. doi: 10.19230/jonnpr.3201

(15) Evangelista LS, Miller PS. Overweight and obesity in the context of heart failure: implications for practice and future research. J
Cardiovasc Nurs. 2006; 21: 27-33.

(16) Conard MW, Haddock CK, Poston WS, Havranek E, McCullough $P$, Spertus J. Impact of obesity on the health status of heart failure patients. ] Card Fail. 2006; 12: 700-6.

(17) Garin O, Soriano N, Ribera A, Ferrer M, Pont A, Alonso J, Permanyer G. Validation of the Spanish Version of the Minnesota Living With Heart Failure Questionnaire. Rev Esp Cardiol. 2008; 61(3): 251-9.

(18) Vasan RS, Larson MG, Benjamin EJ, Evans JC, Levy D. Left ventricular dilatation and the risk of congestive heart failure in people without myocardial infarction. N Engl ] Med. 1997; 336: 1350-5.

(19) World Health Organization. Physical status: the use and interpretation of anthropometry. Report of a WHO Expert Committee. World Health Organ Tech Rep Ser. 1995; 854: 1-452.

(20) Gardin JM, McClelland R, Kitzman D, et al. M-mode echocardiographic predictors of six- to seven-year incidence of coronary heart disease, stroke, congestive heart failure, and mortality in an elderly cohort (the Cardiovascular Health Study). Am J Cardiol. 2001; 87: 1051-7.

(21) Alpert MA. Obesity cardiomyopathy: pathophysiology and evolution of the clinical syndrome. Am J Med Sci. 2001; 321: 225-36

(22) Lind L, Arnlov J, Lampa E. Impact of Aging on the Strength of Cardiovascular Risk Factors: A Longitudinal Study Over 40 Years. JAHA. Vol 1. 2018; 1(1): e7061.

(23) He J, Ogden LG, Bazzano LA, Vupputuri S, Loria C, Whelton PK. Risk factors for congestive heart failure in US men and women: NHANES I epidemiologic follow-up study. Arch Intern Med. 2001; 161: 996-1002

(24) Wilhelmsen L, Rosengren A, Eriksson H, Lappas G. Heart failure in the general population of men -- morbidity, risk factors, and prognosis. J Intern Med. 2001; 249: 253-61.

(25) Guo X, Li ZE. An update on overweight and obesity in rural Northeast China: from lifestyle risk factors to cardiometabolic comorbidities. BMC Public Health. 2014: 1-9.

(26) Kannel WB, Feinleib M, McNamara PM, Garrison RJ, Castelli WP. An investigation of coronary heart disease in families: the Framingham Offspring Study. Am J Epidemiol. 1979; 110: 281-90

(27) Obesity: preventing and managing the global epidemic: report of a WHO consultation. World Health Organ Tech Rep Ser. 2000; 894: 1-253

(28) Poirier P, Giles TD, Bray GA, Hong Y, Stern JS, Pi-Sunyer FX, et-al. Obesity and cardiovascular disease: pathophysiology, evaluation, and effect of weight loss: an update of the 1997 American Heart Association Scientific Statement on Obesity and Heart Disease from the Obesity Committee of the Council on Nutrition, Physical Activity, and Metabolism. Circulation. 2006; 113(6) 898-918.

(29) Kannel WB, Wolf PA, Garrison RJ, eds. The Framingham Study: an epidemiological investigation of cardiovascular disease. Section 34. Some risk factors related to the annual incidence of cardiovascular disease and death using pooled repeated biennial measurements: Framingham Heart Study, 30-year follow-up. Bethesda, Md.: National Heart, Lung, and Blood Institute, 1987. (NIH publication no. 87-2703.)

(30) McKee PA, Castelli WP, McNamara PM, Kannel WB. The natural 
history of congestive heart failure: the Framingham Study. $\mathrm{N}$ Engl ] Med. 1971; 285: 1441-6.

(31) Poirier P. Cardiologists and abdominal obesity: lost in translation? Heart. 2009; 95(13): 1033-5.

(32) Vasan RS, Levy D. Defining diastolic heart failure: a call for standardized diagnostic criteria. Circulation. 2000; 101: 2118-21.

(33) Nagarajan V, Tang WH. Management of comorbid conditions in heart failure: a review. Med Clin North Am. 2012; 96: 975-85.

(34) Mosterd A, Cost B, Hoes AW, et al. The prognosis of heart failure in the general population: the Rotterdam Study. Eur Heart J. 2001; 22: 1318-27.

(35) Sharma R, Von Haehling S, Rauchhaus M, Bolger AP, GenthZotz S, Doehner W, et al. Whole blood endotoxin responsiveness in patients with chronic heart failure: the importance of serum lipoproteins. Eur J Heart Fail. 2005; 7: 479-84.

(36) López-Jiménez F, Cortés-Bergoder M. Obesity and the Heart. Rev Esp Cardiol. 2011; 64(2): 140-9.
(37) Kapoor JR, Heidenreich PA. Obesity and survival in patients with heart failure and preserved systolic function: a U-shaped relationship. Am Heart J. 2010; 159: 75-80.

(38) Pérez-Calvo Jl, Montero-Pérez-Barquero $M$, CamafortBabkowski M, ContheGutiérrez P, Formiga F, Aramburu-Bodas $O$, et al. Influence of admission blood pressure on mortality in patients with acute decompensated heart failure. QJM. 2010; 104: 325-33.

(39) Body Mass Index. Nutrition and food security. World Health Organization Regional Office for Europe [consultado 7 Feb 2020]. Disponible en: www.euro. who.int/nutrition

(40) Zamora E, Lupón J, Urrutia A, González B, Mas D, Pascual T, et al. ¿El índice de masa corporal influye en la moralidad de los pacientes con insuficiencia cardiaca? Rev Esp Cardiol. 2007; 60: $1127-3440$

(41) Arena R, Myers J, Abella J, Pinkstaff S, Brubaker P, Moore B, et al. Influence of etiology of heart failure on the obesity paradox. Am J Cardiol. 2009; 104: 1116-21. 